\title{
LETTER TO THE EDITOR Neuroendocrine prostate carcinoma cells originate from the p63-expressing basal cells but not the pre-existing adenocarcinoma cells in mice
}

\author{
Cell Research (2019) 29:420-422; https://doi.org/10.1038/s41422-019-0149-4
}

\section{Dear Editor,}

The prostate epithelium consists of p63/cytokeratin 14/cytokeratin 5-expressing (p63+/K14+/K5+) basal cells (BCs), K8+/androgen receptor (AR)+ luminal epithelial cells (LECs), and neuroendocrine cells (NECs) expressing synaptophysin (Syp), neural cell adhesion molecule (NCAM) and neuron-specific enolase (NSE). We previously demonstrated that BCs could differentiate into all three cell lineages in the prostate of postnatal mouse. ${ }^{1}$ The LEC-originated AR+ prostate adenocarcinomas are treated with anti-androgen/AR endocrine therapy. However, the AR-negative (AR-) neuroendocrine prostate carcinoma (NEPC) is usually associated with castration resistance, metastasis, resistance to chemotherapy, and poor prognosis. Approximately $2 \%$ of all prostate cancers (PCa) are NEPCs, and $\sim 30 \%$ of adenocarcinomas contain focal NEPC cells after androgen deprivation therapy. ${ }^{2,3}$ Previous studies suggested that NEPC might originate from NECs, ${ }^{4}$ cancer stem cells (CSCs), ${ }^{5}$ or pre-existing adenocarcinoma cells via transdifferentiation. ${ }^{2,6-8}$ However, no lineage tracing study has been performed to test whether NEPC can arise from BCs without transdifferentiation.

To examine whether NEPC cells arise from p63+ BCs, we generated p63-CreERT2;Rosa-LoxP-STOP-LoxP-tdRFP;TRAMP (designated as p63iCre;iRFP;TRAMP) mice by breeding p63iCre;iRFP and TRAMP mice (Supplementary information, Data S1). ${ }^{1,9}$ TRAMP mice are prone to develop both adenocarcinomas and NEPCs, but the cell of origin for NEPC is unknown. ${ }^{9}$ In the TRAMP mouse prostate, SV40 $\mathrm{T} / \mathrm{t}$ antigens start expressing at 3 weeks of age. Low-grade hyperplasia and prostate intraepithelial neoplasia (PIN) develop at 4 and 12 weeks of age, respectively. ${ }^{9}$ In p63iCre;iRFP;TRAMP mice, tamoxifen treatment-activated CreERT2 triggers irreversible red fluorescent protein (RFP) expression in BCs, which is an ideal model for lineage tracing. p63iCre;iRFP;TRAMP mice were treated with tamoxifen during 2-3 weeks of age, which resulted in RFP expression in $70.6 \%$ of p63 + BCs and none of LECs at 4 weeks of age (Fig. 1a). All tamoxifen-treated mice developed large NEPCs and prostate adenocarcinomas by 20 weeks of age. On the adjacent tumor sections, the tumor cells, NEPC cells and RFP + cells were tested by immunohistochemistry (IHC) for SV40 T antigen, Syp and RFP. SV40T antigen was expressed in both Syp+ NEPC cells and Sypadenocarcinoma cells, although its immunoreactivity in NEPC cells was much higher than that in adenocarcinoma cells. Results from IHC for other NEC markers including NCAM and NSE were consistent with the IHC results for Syp (data not shown). We found that tumor areas with either Syp+ NEPC or Syp- adenocarcinoma had many RFP + tumor cells, indicating that both NEPC and adenocarcinoma cells can originate from p63-expressing BCs (Fig. 1b). Triple immunofluorescent (IF) staining confirmed the Syp+/RFP+/SV40T + NEPC cells and the Syp-/RFP+/SV40T+ adenocarcinoma cells. Double IF staining for K14 and RFP or K8 and RFP detected large numbers of $\mathrm{K} 14+/ \mathrm{RFP}+\mathrm{BCs}$ and $\mathrm{K} 8+/ \mathrm{RFP}+$ adenocarcinoma cells.
At this 20 -week stage, $45.5 \%$ of the total Syp+/SV40T+ NEPC areas consisted of Syp+/RFP+/SV40T+ NEPC cells, $99.5 \%$ of the K14+ BCs were RFP $+/ \mathrm{K} 14+$ cells, and $92 \%$ of the K8+ adenocarcinoma cells were RFP $+/$ K8 + cells (Supplementary information, Fig. S1a-c). The development of RFP-negative NEPC cells, BCs and adenocarcinoma cells might be a result of incomplete RFP labeling of p63+ BCs in TRAMP mice during 3-4 weeks of age. These results demonstrate that p63+ BCs labeled with RFP are the cells of origin for NEPC, adenocarcinoma and BCs in TRAMP mice.

To examine whether K8+ LECs or pre-existing K8+ adenocarcinoma cells could transdifferentiate into NEPC cells, we generated K8-CreERT2;Rosa-LoxP-STOP-LoxP-tdRFP;TRAMP (designated as K8iCre;iRFP;TRAMP) mice and traced the K8+ cell lineage. We treated these mice with tamoxifen during 2-3 weeks of ages and found that $54.5 \%$ of the K8+ LECs and adenocarcinoma cells in the prostates were labeled with RFP (Fig. 1c). To our surprise, no Syp+/RFP+/SV40T + NEPC cells were detected in the prostate tumors containing both large and small Syp+/SV40T+ NEPC regions at 20 weeks of age, although Syp-/RFP+/SV40T+ adenocarcinoma cells were detected (Fig. 1d). These results indicate that NEPC cells are not derived from K8+ LECs and adenocarcinoma cells, different from the results obtained from the double p53 and Pten knockout-induced PCa mouse model in which all NEPC cells were transdifferentiated from LEC lineage. ${ }^{8}$ This difference indicates that NEPC can originate from transformed p63+ progenitor cells without pre-differentiation into LEC lineage in one PCa subtype or from transformed LEC lineage in another PCa subtype.

Interestingly, the ratios of Syp-/RFP+/SV40T+ to Syp-/RFP-/ SV40T+ adenocarcinoma cells and LECs were reduced from $54.5 \%$ at 4 weeks of age to $17.2 \%$ at 20 weeks of age in K8iCre;iRFP; TRAMP mice (Supplementary information, Fig. S1d), which could be attributed to loss of RFP + adenocarcinoma cells labeled at 4 weeks of age and gain of RFP- adenocarcinoma cells derived from unlabeled basal progenitor cells during tumor progression. This explanation is consistent with the observation that p63+ BCs are capable of differentiating into AR+ LECs and adenocarcinoma cells in the prostates of TRAMP mice.

The rat NRP-152 cell line contains prostate epithelial progenitor cells. ${ }^{10}$ To test whether these progenitor cells could differentiate into NEPC cells, we generated SV40 T/t-expressing NRP-152 cell lines and examined their cellular markers. In the newly established clones cultured for 3 weeks, NSE was not expressed in the control cells harboring the empty vector. NSE was expressed in clone T6 at a high level and clones T8, T9 and T10 at low levels. AR and p63 were present in the control cells; their expression levels nearly disappeared in clone T6 but only slightly decreased in clones T8, T9 and T10. K14 expression was similar in all clones (Fig. 1e). These results demonstrate that clones T8, T9 and T10 co-express SV40T with basal or progenitor cell markers p63 and K14 and low-level 

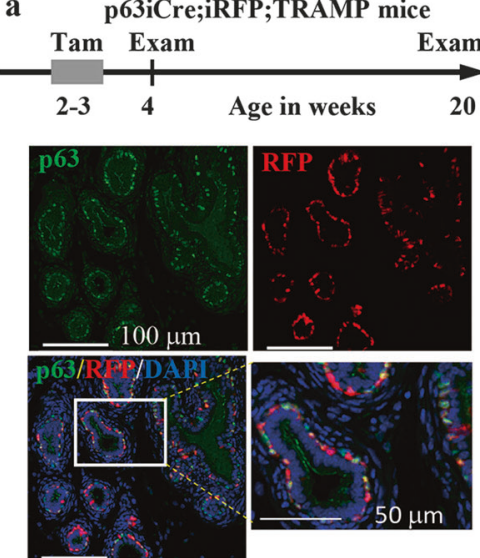

RFP+/p63+: $70.6 \pm 2.5 \%$
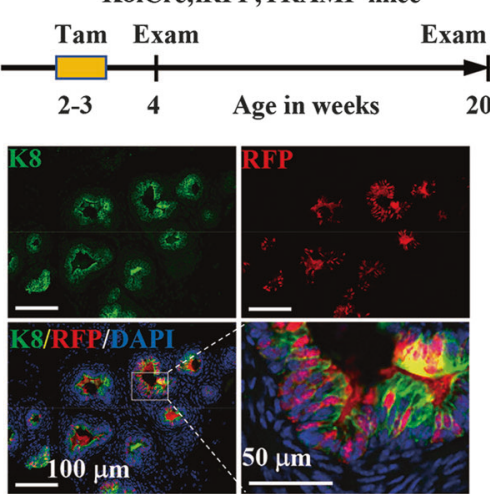

RFP+/K8+: $54.5 \pm 2.8 \%$
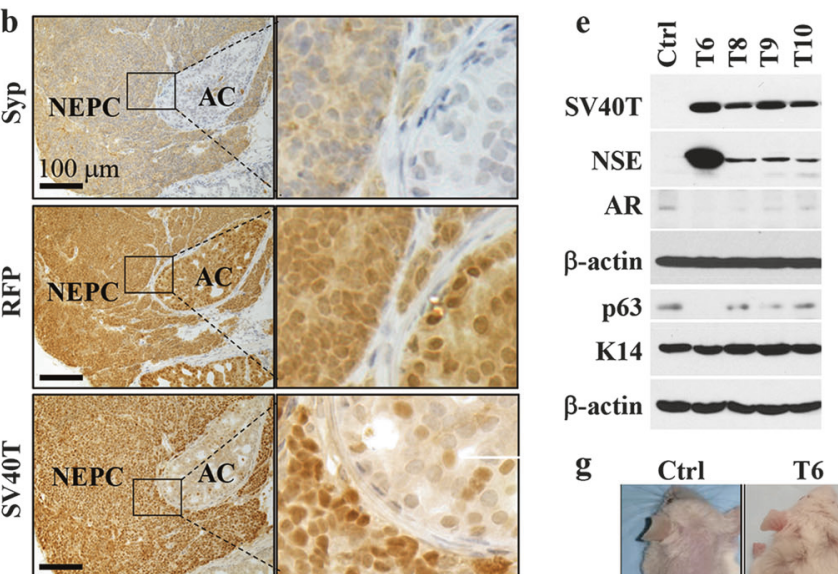

f
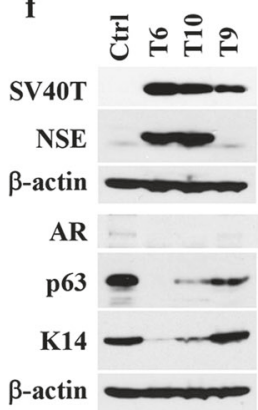

g

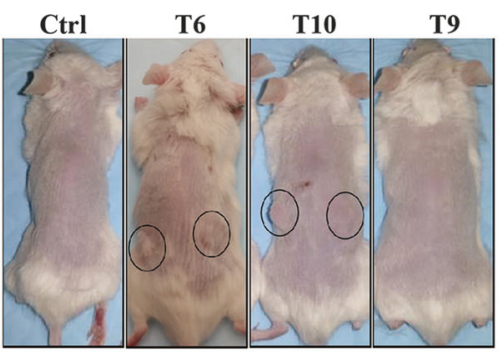

h
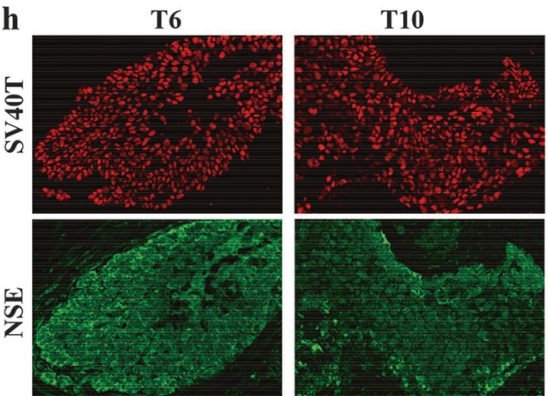

Fig. 1 NEPC cells originate from p63+ basal progenitor cells but not LECs or pre-existing adenocarcinoma cells. a Experimental procedure and representative double immunofluorescent (IF) staining for p63 and RFP on prostate sections at 4 weeks of age. p63iCre;iRFP;TRAMP mice ( $n=$ 6) were treated with tamoxifen (Tam) during 2-3 weeks of age and examined for RFP-labeling efficiency of p63+ BCs at 4 weeks of age, and for RFP-labeled NEPC cells, BCs and adenocarcinoma cells at 20 weeks of age (Supplementary information, Fig. S1a-c). At 4 weeks, $70.6 \%$ of the examined $\mathrm{p} 63+\mathrm{BCs}(n=921)$ were RFP positive. The boxed area is enlarged and shown on the right. $\mathbf{b}$ Immunohistochemistry (IHC) for Syp, RFP and SV40T on adjacent serial prostate tumor sections at 20 weeks of age. NEPC, neuroendocrine prostate carcinoma; AC, adenocarcinoma. c Experimental procedure and representative double IF staining for K8 and RFP on prostate sections at 4 weeks of age. K8iCre;iRFP;TRAMP mice $(n=8)$ were treated with Tam during 2-3 weeks of age, and analyzed for RFP-labeling efficiency of K8+ LECs at 4 weeks of age $(n=3)$, and for RFP-labeled NEPC and adenocarcinoma cells at 20 weeks of age ( $n=5 ;$ Supplementary information, Fig. S1d). At 4 weeks, $54.5 \%$ of the counted K8+ LECs and adenocarcinoma cells $(n=2,285)$ were RFP positive. The boxed area is enlarged and shown on the right. d Representative images for Syp, RFP and SV40T IHC on adjacent serial prostate tumor sections at 20 weeks of age. e, $\mathbf{f}$ Western blot analysis of SV40T, NSE, AR, p63 and K14 in cloned NRP-152 cell lines transduced by control (Ctrl) or SV40 T/t-expressing lentivirus (other lanes). The cloned cells examined in e, $\mathbf{f}$ were cultured for 3 weeks and one year, respectively. $\beta$-actin served as a loading control. $\mathbf{g}$ Xenograft tumor growth assay in SCID mice. Tumors formed from clones T6 and T10 are circled. Control and T9 cells did not develop any tumors. The images are representative of 5 mice in each group. $\mathbf{h}$ Representative double IF staining for SV40 T antigen (red) and NSE (green) on tissue sections prepared from xenograft tumors derived from clones T6 and T10 in SCID mice

neuroendocrine marker NSE, while clone T6 co-expresses SV40T with only K14 and high-level NSE at this early time point, suggesting that clones T8, T9 and T10 are in their early intermediate state and clone T6 is in its late intermediate state toward NEPC differentiation. During the subsequent passages, clone T8 was lost due to failure of continuous growth. After oneyear in culture, control cells maintained their expression levels of all proteins examined at both early and late time points (Fig. 1f). Of note, the stronger p63 band from control cells shown in Fig. If was a result of enhanced experimental conditions for detecting weak p63 bands from other clones. In comparison with the control cells, clone T6 maintained similar expression profiles of NSE, AR, and p63 but reduced K14 expression to a shallow level, and clone 10 showed robustly increased NSE expression and decreased AR, p63 and K14 expression (Fig. 1f). These results indicate that these two clones have gone much farther toward NEPC differentiation in terms of losing progenitor cell markers and gaining the neuroendocrine marker. In contrast, clone T9 expressed lower level of SV40T compared to the other two clones, became NSE negative, and retained AR, p63 and K14 expression, indicating that this clone has reversed its partial NEPC differentiation and returned to its progenitor state (Fig. 1f). These results suggest that SV40 T/t overexpression beyond a threshold is sufficient to induce NEPC differentiation from a subpopulation of NRP-152 cells in culture.

To test whether SV40 T/t-expressing NRP-152 cells could develop NEPC in vivo, we subcutaneously injected these cells into male SCID mice. The control cells were unable to form any tumors. The cells of clone T9 without NSE expression but with AR, SV40 T/t, p63 and K14 expression were also incapable of developing any tumors. Interestingly, the cells of clone T6 and clone $\mathrm{T} 10$ developed palpable tumors within 4 weeks after being 
inoculated (Fig. 1g). When examined at week 8 after inoculation, the tumor cells derived from either clone T6 or clone T10 showed both SV40T and NSE expression (Fig. 1h), and the majority of these tumor cells were K14 negative (Supplementary information, Fig. S2a). In summary, T6 and T10 clones with SV40 T/t expression developed NEPC in vivo.

We previously showed that $\mathrm{p} 63+\mathrm{BCs}$ could differentiate into prostate neuroendocrine cells in normal mice, ${ }^{1}$ and also identified the rare subsets of $\mathrm{p} 63+/ \mathrm{Syp}+$ and $\mathrm{K} 5+/ \mathrm{SV} 40 \mathrm{~T}+/ \mathrm{Syp}+$ cells in TRAMP mice with prostate-specific steroid receptor coactivator-3 $(S R C-3)$ knockout. $^{11}$ In the present study, we demonstrated that overexpression of SV40 T/t, which inhibits p53, RB and PP2A tumor suppressor functions, can induce prostate progenitor cells to differentiate into NEPC cells via early and late intermediate states with an expression of both neuroendocrine cell marker and basal/ progenitor cell markers. Based on these findings, we propose a progenitor-initiating NEPC model. At early PCa-initiating stages, transformed intermediate progenitor cells deficient of p53, RB and PP2A may preferentially differentiate into adenocarcinoma progenitor cells but also produce a small number of NEPC cells. During PCa progression stages, the adenocarcinoma progenitor cells continuously produce adenocarcinoma cells, and the matured adenocarcinoma cells are continuously lost over time to constitute a dynamic change in the adenocarcinoma cell population. However, the rare NEPC cells at early stages gradually increase their number by self-proliferation and eventually become a large cell population in advanced prostate tumors (Supplementary information, Fig. S2b). This model suggests that NEPC cells can arise independently without transdifferentiation from the preexisting adenocarcinoma cells and thereby should be diagnosed and treated as early as possible instead of waiting for the completion of endocrine therapy.

\section{ACKNOWLEDGEMENTS}

We thank Souling Zhou for experimental assistance and Dr. Norman Greenberg for providing TRAMP mouse line. This work was partially supported by NIH grants (CA193455 and DK113954), and Cancer Prevention and Research Institute of Texas grants (RP150197 and RP170330).

\section{AUTHOR CONTRIBUTIONS}

D.L. and J.X. designed the study. D.L., Y.L. and L.L. performed experiments. D.L. analyzed data. D.D. generated NRP-152 cells. D.L., W.L., D.D. and J.X. wrote or revised the manuscript. J.X. directed the study.

\section{ADDITIONAL INFORMATION}

Supplementary information accompanies this paper at https://doi.org/10.1038/ s41422-019-0149-4.

Competing interests: J. Xu is a shareholder of Coactigon, Inc. The remaining authors declare no competing interests.

Dong-Kee Lee ${ }^{1}$, Yonghong Liu ${ }^{1,2}$, Lan Liao ${ }^{1}$, Wenliang $\mathrm{Li}^{3}$, David Danielpour $\mathbb{D}^{4}$ and Jianming $\mathrm{Xu}^{1}$

${ }^{1}$ Department of Molecular and Cellular Biology, and Dan L. Duncan Comprehensive Cancer Center, Baylor College of Medicine, One Baylor Plaza, Houston, TX 77030, USA; ${ }^{2}$ Institute of Biosciences and Technology, Texas A\&M University Health Science Center, Houston,

TX 77030, USA; ${ }^{3}$ Texas Therapeutics Institute, Brown Foundation

Institute of Molecular Medicine, University of Texas Health Science Center at Houston, Houston, TX 77030, USA and ${ }^{4}$ Division of General Medical Sciences-Oncology, and Department of Pharmacology, Case Western Reserve University School of Medicine, Cleveland, $\mathrm{OH} 44106$, USA

Correspondence: Jianming Xu (jxu@bcm.edu)

\section{REFERENCES}

1. Lee, D. K., Liu, Y., Liao, L., Wang, F. \& Xu, J. Int J. Biol. Sci. 10, 1007-1017 (2014).

2. Beltran, H. et al. Clin. Cancer Res 20, 2846-2850 (2014).

3. Small, E. J. et al. J. Clin. Oncol. 33, 5003 (2015).

4. Hu, Y., Ippolito, J. E., Garabedian, E. M., Humphrey, P. A. \& Gordon, J. I. J. Biol. Chem. 277, 44462-44474 (2002).

5. Palapattu, G. S. et al. Prostate 69, 787-798 (2009).

6. Svensson, C. et al. Nucleic Acids Res 42, 999-1015 (2014).

7. Zhu, Y. et al. Prostate 74, 1086-1094 (2014).

8. Zou, M. et al. Cancer Discov. 7, 736-749 (2017).

9. Greenberg, N. M. et al. Proc. Natl. Acad. Sci. USA 92, 3439-3443 (1995).

10. Danielpour, D. J. Cell Sci. 112, 169-179 (1999).

11. Tien, J. C. et al. Int J. Biol. Sci. 10, 1116-1127 (2014). 